\title{
Temperature-Dependent Internal Quantum Efficiency of Blue High-Brightness Light- Emitting Diodes
}

\author{
Ilya E. Titkov, Sergey Yu. Karpov, Amit Yadav, Vera L. Zerova, Modestas Zulonas, Bastian Galler, \\ Martin Strassburg, Ines Pietzonka, Hans-Juergen Lugauer, and Edik U. Rafailov, Senior Member,
}

IEEE

\begin{abstract}
Internal quantum efficiency (IQE) of a blue highbrightness InGaN/GaN LED was evaluated from the external quantum efficiency measured as a function of current at various temperatures ranged between $13 \mathrm{~K}$ and $440 \mathrm{~K}$. Processing the data with a novel evaluation procedure based on the ABC-model, we have determined the temperature dependent IQE of the LED structure and light extraction efficiency of the LED chip. Separate evaluation of these parameters is helpful for further optimization of the heterostructure and chip designs. The data obtained enable making a guess on the temperature dependence of the radiative and Auger recombination coefficients, which may be important for identification of dominant mechanisms responsible for the efficiency droop in III-nitride LEDs. Thermal degradation of the LED performance in terms of the emission efficiency is also considered.
\end{abstract}

Index Terms - Light-emitting diodes, III-nitrides, internal quantum efficiency, light extraction efficiency, temperature dependence, Auger recombination, optical emission spectra

\section{INTRODUCTION}

$\mathrm{T}$ HE use of state-of-the-art light emitting diodes as the sources for solid-state lighting requires ever increasing optical power emitted from the unit area of the devices. In the case of III-nitride LEDs, that is a real challenge because of the efficiency droop, i.e. decrease of the LED emission efficiency with operating current, specific for this kind of light emitters and limiting their performance [1,2]. Generally, the droop is attributed to both thermal and non-thermal mechanisms. The former ones refer to the interplay of temperature-dependent radiative and non-radiative recombination channels in the LED structures and, in some particular cases, to the carrier

This work was supported by European Union FP7, NEWLED project, Grant number 318388

Ilya E. Titkov, Amit Yadav, Vera L. Zerova, Modestas Zulonas and Edik Rafailov are with the School of Engineering and Applied Science, Aston Institute of Photonic Technologies, Aston University, Birmingham, B47ET, UK (e-mail: i.titkov@aston.ac.uk).

Sergey Yu. Karpov is with STR Group - Soft-Impact., Ltd, P.O.Box 83, 194156 St.Petersburg, Russia (e-mail: sergey.karpov@ str-soft.com).

Bastian Galler, Martin Strassburg, Ines Pietzonka and Hans-Juergen Lugauer are with OSRAM Opto Semiconductors GmbH, CTO Advanced Concepts \& Engineering, Novel Technologies, Leibnitzstr., 93055 Regensburg, Germany (e-mail: ines.pietzonka@osram-os.com). leakage from the active regions. Despite the thermal droop can be suppressed to some extent by reducing the LED thermal resistance via proper chip design, it remains, nevertheless, an important factor in view of the possibility of III-nitride LEDs to operate effectively at elevated temperatures. Variety of mechanisms has been invoked for explanation of the nonthermal droop (see, e. g., their review given in [3]), though Auger recombination enhanced with current seems to be the most likely one, as suggested in [4-6] and justified by recent experiments [7-9]. Consideration of the Auger recombination as the dominant mechanism of the efficiency reduction with current, has enabled generation of a number of approaches to improve the LED performance, like the use of a wide singlequantum well (SQW) or a closely coupled multi-quantum well (MQW) active region, tailoring composition and doping of the barriers separating QWs, etc. (see, in particular, [3] for a more detailed discussion of the approaches). One more contribution to the efficiency droop comes from the current crowding resulting in localization of the photon emission region under metallic electrodes that cover emitting surfaces of some types of vertical LEDs [10]. This effect, leading to reduction of the light extraction efficiency (LEE) with operating currents, can be largely suppressed by using advanced chip designs like thin-film flip-chips proposed by Lumileds [11] or UX:3 chips developed by Osram Opto Semiconductors [12].

Nowadays, understanding of all the possible reasons for the efficiency droop is quite critical to find ways for further improvement of III-nitride LED performance in terms of the commonly measured external quantum efficiency $\eta_{e}$ (EQE) as a function of operating current. Being the product of the internal quantum efficiency $\eta_{i}$ and light extraction efficiency $\eta_{\text {ext }}$ at negligible carrier leakage from the active region, EQE provides an integral information on the recombination and photon emission processes in the LED. Separate determination of IQE and LEE would be much more helpful, providing correlation between these parameters and specific design of either LED heterostructure or LED chip, respectively. Therefore, development of the techniques for separate evaluation of IQE and LEE is in the focus of researches for a long time.

To date, efficiency of light extraction from LED dice is determined mainly theoretically. The most popular methods 
applied for this purpose are the ray tracing and the finitedifference time-domain (FDTD) modeling [13]. The former approach is quite suitable to account for properties of the photon ensemble produced in the LED die. However, in its conventional formulation, the ray tracing ignores some factors related to photon polarization and neglects diffraction effects important in the case of textured surfaces used for enhancement of light extraction. The later drawback is absent in the FDTD approach operating with the rigorous Maxwell's equations. However, the method is generally time- and computer resource-costly. Therefore, it normally operates with artificial boundary conditions, leading to uncertain inaccuracy of the results obtained. In addition, FDTD method considers a limited number of emitting dipoles, their polarizations, and local positions inside the LED active region, thus oversimplifying the description of the photon ensemble properties. So, the ray tracing and FDTD simulations, as well as other approaches reviewed in [13], cannot provide at the moment estimates for LEE with an a priori known accuracy.

Temperature-dependent variable-excitation photoluminescence (PL) $[14,15]$ and temperature-dependent electroluminescence (TDEL) $[16,17]$ are the technique most widely used for evaluation of IQE. They are based on intuitive assumptions that (i) LEE does not depend on temperature and (ii) nonradiative recombination can be neglected at low temperatures, providing nearly $100 \%$ IQE. Since LEE is affected by light absorption inside the LED die and, in particular, by freecarrier absorption in the contact layers, the former assumption seems to be generally incorrect because of thermal activation of donors and acceptors. As for the latter assumption, the data reported in $[16,17]$ clearly show that IQE may approach a maximum value only in a limited range of the current variation, i.e. at other currents, IQE is definitely less than $100 \%$ even at very low temperatures. So, the latter assumption is still waiting for its reliable experimental justification.

Recently the use of the ABC-recombination model for IQE evaluation has become popular. Already the earlier paper [4], reported on PL study of the emission efficiency droop in InGaN, has shown the ABC-model to fit quite well the dependence of the efficiency on the excitation power density. On the basic of the model, Ryu et al., have derived a transcendent equation for IQE as a function of current/current density with only two free parameters: maximum IQE value $\eta_{i}^{\max }$ and current/current density corresponding to this maximum [18]. The latter parameter could be found directly from the measured $\mathrm{EQE}$ dependence on the operating current $I$, whereas the former one could be obtained by fitting the theoretical $\eta_{e}(I)$ curve to the measurements. Such a method has two main drawbacks. Firstly, it requires numerical solution of the transcendent equation to determine theoretical dependence $\eta_{i}(I)$. Secondly, this method produces a large error being applied to the data with systematic deviation of the $\eta_{e}(I)$ behavior from that predicted by the ABC-model (see discussion on the deviation given in [19]).

Attempts to develop a practical technique for the IQE evaluation, which would not implement any computational stage, has resulted in proposal of two approximate versions of the approach [18]. One of them suggested to measure the width of the dome-like $\eta_{e}(I)$ dependence at a certain height [20], whereas another one implied to measure the curvature of the dependence at the maximum point [21], which enabled estimation of the peak IQE. Despite the attractive simplicity of the approaches, they were not yet widely employed in practice.

Following the procedure developed for conventional III-V compounds [22], the peak IQE value and LEE of III-nitride LEDs were estimated from the low-signal behavior of the measured $1 / \eta_{e}$ as a function of the square root of the output optical power $P_{\text {out }}$ [23]. Unfortunately, just at low currents (optical powers) remarkable deviation of the EQE behavior from that predicted by the ABC-model was observed, at least in the case of green LEDs [6], which could be attributed to carrier localization due to compositional fluctuations in InGaN QWs [24]. On the other hand, the impact of the carrier localization on EQE of blue LEDs is expected to be somewhat weaker.

In this paper, we will show that the approach $[22,23]$ can be easily extended to the whole range of the current/optical power variation, providing an express tool for separate evaluation of the peak IQE and LEE. Using the extended approach, we have studied the evolution of the high-brightness blue LED efficiency with temperature in the wide range of its variation, $13-440 \mathrm{~K}$.

The paper is organized as follows. Section II describes the samples used in our study, the experimental techniques applied, and the suggested procedure of data processing aimed at separate estimating LEE and peak IQE value. Detailed data of the measurements are presented in Sec.III. Discussion on the data and physical mechanisms controlling the temperature evolution of the LED efficiency is given in Sec.IV. Section V summarizes the obtained results and conclusions made and identifies still open questions.

\section{SAMPLES, EXPERIMENTS, AND DATA PROCESSING}

\section{A. Samples}

We have studied high-brightness InGaN-based blue LEDs fabricated at Osram Opto Semiconductors. The LED structures were grown by Metalorganic Chemical Vapor Deposition on (0001)-sapphire substrates. All the structures consisted of an undoped $\mathrm{GaN}$ layer followed by a Si-doped nGaN contact layer, undoped InGaN/GaN MQW active region, and a Mg-doped $\mathrm{p}$-GaN contact layer. Highly reflective electrodes were formed to both contact layers of the LED structure. The structures were processed as UX:3 chips [12] and mounted into the Golden Dragon packages without molding with silicone and forming lenses. The absence of any media adjacent to the chip surface was essential for correct temperature- and intensity-dependent electroluminescence (TIDEL) measurements. On the other hand, LEE of such samples is lower than in common applications. 


\section{B. Experimental}

As a basic experimental method we have employed T-IDEL [17]. A Labsphere CDS-600 spectrometer and helium closedcycle cryostat Janis CCS-450 were used for this purpose. In order to determine EQE, we measured the electroluminescence (EL) intensity at various temperatures from 13 to 440 $\mathrm{K}$ in a wide range of operating currents, from $10^{-8}$ to $0.8 \mathrm{~A}$. To cover a large range of the measured radiant fluxes, from $1 \mathrm{nW}$ to $0.8 \mathrm{~W}$, we used variation of the photodetector exposure time from $1 \mathrm{~ms}$ to $5 \mathrm{~s}$ and ND1-4 filters for light intensity attenuation. EQE was measured first in the integrating sphere and then in the cryostat at room temperature (RT); after that the optical alignment was not changed at other temperatures. In order to obtain absolute values of $\mathrm{EQE}$ for temperatures other than RT, we used the normalization factors calculated for the RT. The cryostat position was tuned every time at other temperatures to compensate thermo shift of the sample holder. Therefore, the optical alignment including absolute sample position was not change during all the TDEL measurements.

To avoid the effect of LED self-heating on the EQE droop at the currents higher than $\sim 50-70 \mathrm{~mA}$, we used $20 \mathrm{~ms}$ current pulses at a low duty cycle. The absence of the self-heating was confirmed by negligible red shift of the emission spectra and their broadening with current.

The current-voltage (I-V) characteristics of the LEDs were measured by the Keithley 4200 semiconductor characterization system in the temperature range of 13-440 K.

\section{Data processing}

EQE of an LED is determined from the measured lightcurrent characteristic $P_{\text {out }}(I)$, using the relationship

$\eta_{e}=q P_{\text {out }} / I \hbar \omega$,

where $q$ is the electron charge and $\hbar \omega$ is the photon energy averaged over the emission spectrum. Following [22,23,25], we will plot EQE as a function of $P_{\text {out }}$ and find in this plot the peak EQE value $\eta_{e}^{\max }$ and the power $P_{\max }$ corresponding to this peak. Then the normalized optical power $p=P_{\text {out }} / P_{\max }$ is calculated, and the $\eta_{e}(p)$ dependence is derived from the measurements.

Assuming the absence of the electron leakage in the LED structure, which has been justified experimentally [26], and using the $\mathrm{ABC}$-model one can obtain the analytic expression for $\mathrm{EQE}$ as a function of the normalized optical power:

$\eta_{e}(p)=\eta_{e x t} \eta_{i} \quad, \quad \eta_{i}=\frac{Q}{Q+p^{1 / 2}+p^{-1 / 2}}$

with $Q$ being the only dimensionless parameter determining the shape of the $\eta_{e}(p)$ dependence. This parameter was introduced in [25] and called 'quality factor' in [27] due to its relation to the peak IQE value: $\eta_{i}^{\max }=Q /(Q+2)$. On the other hand, $Q=B /(A C)^{1 / 2}$ is the combination of the Shockley-Read-Hall recombination constant $A$, the radiative recombination constant $B$, and the Auger recombination constant $C$. As it follows from (2), the ratio $\eta_{e}^{\max } / \eta_{e}(p)=\eta_{i}^{\max }+\frac{p^{1 / 2}+p^{-1 / 2}}{Q+2}$

depends linearly on the combination $p^{1 / 2}+p^{-1 / 2}$ and provides the value of $\eta_{i}^{\max }$ by extrapolation of the linear dependence to $p^{1 / 2}+p^{-1 / 2} \rightarrow 0$. One can see that such a linearity should be met at any value of $p$, i.e. in the whole range of the current/optical power variation, if the experimental dependence $\eta_{e}(p)$ can be fitted well by the ABC-model. So, considerable deviation from the linear dependence (3) may serve as an indicator of the model inapplicability to interpret the data on $\mathrm{EQE}$.

Fitting the experimental $\eta_{e}^{\max } / \eta_{e}(p)$ points by a line enables also estimation of the $Q$-factor via the slope of the line. This improves remarkably the accuracy of the peak IQE determination due to the relationship between $\eta_{i}^{\max }$ and the $Q$ factor mentioned above. As soon as $\eta_{i}^{\max }$ is found, LEE can be calculated as follows: $\eta_{e x t}=\eta_{e}^{\max } / \eta_{i}^{\max }$.

\section{RESULTS}

This section summarizes and discusses the data of measurements obtained by the techniques described in Sec.IIB. The results of processing the data on EQE as a function of operating current are given here as well.

\section{A. Current-voltage characteristics}

Figure 1 shows the I-V characteristics of the LED obtained at various temperatures. One can see the examined blue LED to demonstrate diode-like I-V curves even at temperatures as low, as $13-50 \mathrm{~K}$, which is in line with the previous reports (see, e.g. [28]).

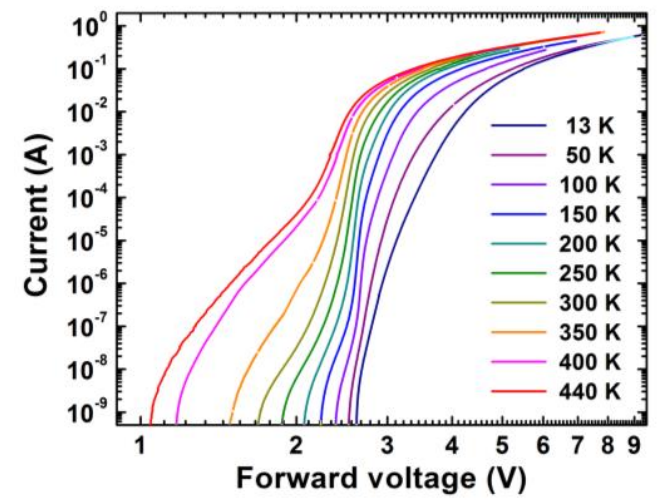

Fig. 1. Current-voltage characteristics of blue LED measured at various temperatures

Every I-V curve plotted in Fig.1 contains two sections: lowcurrent and high-current ones. The low-current section corresponds to the forward voltages typically less than $\sim 2.2 \mathrm{~V}$. It can be attributed to either trap-assisted tunneling of electrons and holes through some midgap electronic states or carrier leakage via extended defects like threading dislocations, micropipes, and V-defects. The high-current section of an I-V characteristic is normally observed at the voltages greater than $\sim 2.7 \mathrm{~V}$ and is associated with the carrier 
injection into the LED active region. The change in the slope of the I-V curve in this section is caused by contributions of the p-n junction resistance dependent on current and the LED series resistance. Estimates made by fitting an experimental I$\mathrm{V}$ curve with the Shockley's diode equation corrected to account for the series resistance of the diode have provided the resistance to decrease nearly linearly from $7.1 \Omega$ at $13 \mathrm{~K}$ to 6.0 $\Omega$ at $440 \mathrm{~K}$. The small variation of the LED series resistance in the temperature range of $13-440 \mathrm{~K}$ is the evidence for (i) the absence of the remarkable carrier freezing in the contact layers and (ii) the fact that $\mathrm{n}$ - and especially p-electrodes formed to the contact layers keep their ohmic properties in the whole temperature range examined.

If the low-current sections of the I-V characteristics are related to the trap-assisted tunneling of electrons and holes or carrier leakage through the extended defects, they do not contribute to the radiative recombination of electrons and holes in the active region, thus forming the channel of extra carrier losses that are not accounted for by the ABC-model. The potential impact of the losses on the results of the EQE data processing is discussed in detail in Sec.IVA.

\section{B. Optical emission spectra}

Figure 2 shows the EL spectra of the LED at various temperatures measured at the operating current of $3 \mathrm{~mA}$ and corresponding to either maximum or plateau in the $\mathrm{EQE}$ dependence on current in the temperature range of 13-300 K. The spectra consist of a main band-to-band transition peak and two phonon replicas, especially pronounced at low temperatures. The observation of the phonon replicas is the evidence of high quality of the MQW active region in the LED structure. At higher temperatures, however, the peaks related to the phonon replicas merge with the main one, forming extended long-wavelength tails in the spectra. The shortwavelength wings of the spectra become larger with temperature, reflecting evolution of the carrier population in the conduction and valence bands.

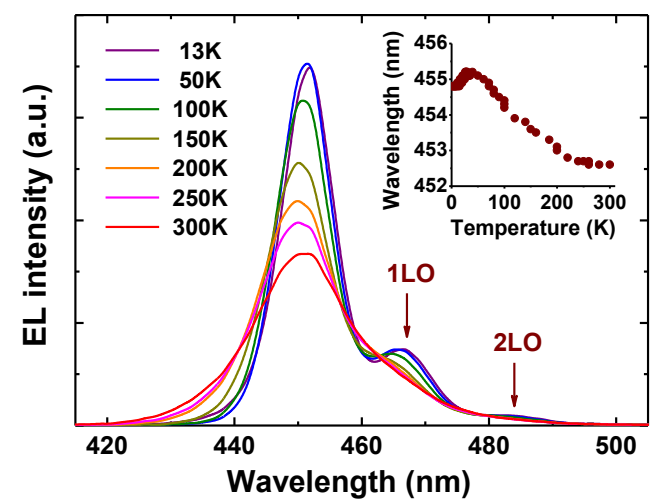

Fig. 2. Emission spectra of blue LED measured at various temperatures. Arrows indicate the spectral position of phonon replicas. Inset shows the variation of the mean emission wavelength with temperature.

The mean emission wavelength, i.e. that corresponding to the mean photon energy, is found to be quite stable with temperature: it shifts by only $\sim 3 \mathrm{~nm}$ in the whole range of 13-
$300 \mathrm{~K}$. Very similar spectra were obtained by PL using the resonant excitation of the LED structure with a violet laser diode emitting at $405 \mathrm{~nm}$ (not shown here). In both EL and PL emission spectra, the emission wavelength exhibited a weak Sshaped dependence on temperature commonly attributed to the carrier localization in InGaN/GaN MQWs [29].

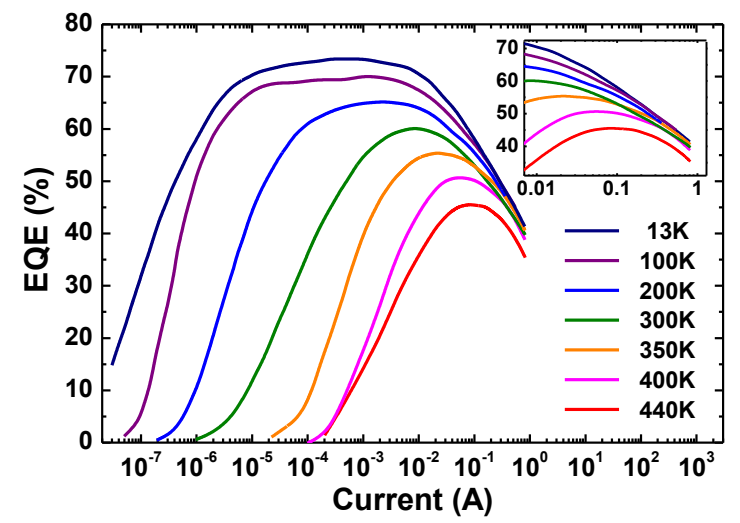

Fig. 3. EQE as a function of LED operating current measured at various temperatures. Inset displays the high-current behavior of EQE in more detail.

\section{External quantum efficiency}

Figure 3 displays selected EQE curves as a function of current measured in the wide range of temperatures, from 13 $\mathrm{K}$ to $440 \mathrm{~K}$. The EQE behavior at high currents is zoomed in the inset of the figure. All the curves exhibit a dome-like shapes with the width gradually increasing under temperature lowering. The increase in the widths correlates with the rise of the EQE maximum value. Note that all the curves tend to merge at high operating currents without intersection. This contrasts remarkably with the results reported in [30] for a blue LED fabricated by Nichia where the onset of the efficiency droop shifted dramatically to low currents at lower temperatures, resulting in intersection of various EQE curves.

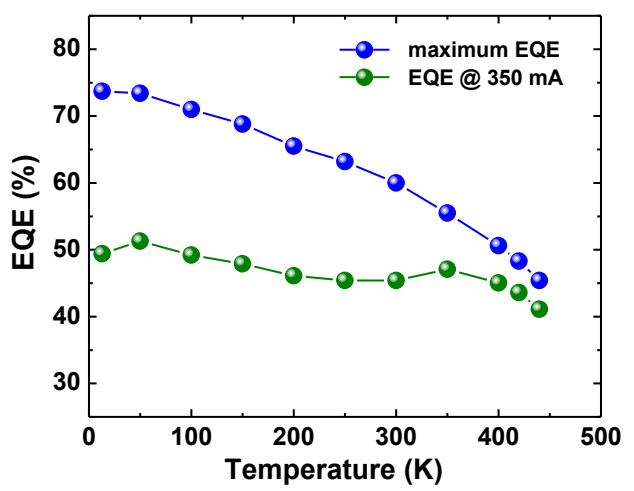

Fig. 4. Maximum $\mathrm{EQE}$ and $\mathrm{EQE}$ measured at the operating current of 350 $\mathrm{mA}$ as a function of temperature.

Dependence of the measured maximum EQE value on temperature is shown in Fig.4. The EQE maximum decreases with temperature from $73.7 \%$ at $13-50 \mathrm{~K}$ to $45.4 \%$ at $440 \mathrm{~K}$. In contrary, the $\mathrm{EQE}$ value corresponding to the current of 350 $\mathrm{mA}$ depends on temperature rather weakly. It decreases from $\sim 49-51 \%$ at $13 \mathrm{~K}$ to $\sim 41 \%$ at $440 \mathrm{~K}$. Such a behavior is 
discussed in more detail in Sec.IVC.

\section{Results of data processing}

The processing procedure described in Sec. IIC has been applied to the data obtained. Figure 5 shows the processing results for selected temperatures of 13,300 , and $440 \mathrm{~K}$. One can see that the experimental $\eta_{e}^{\max } / \eta_{e}(p)$ ratios depends on the variable $Y=p^{1 / 2}+p^{-1 / 2}$ quite linearly up to the values of $\sim 30-40$. Small $Y$, close to 2, correspond to EQEs next to its maximum, whereas large $Y$, associated with either high or low
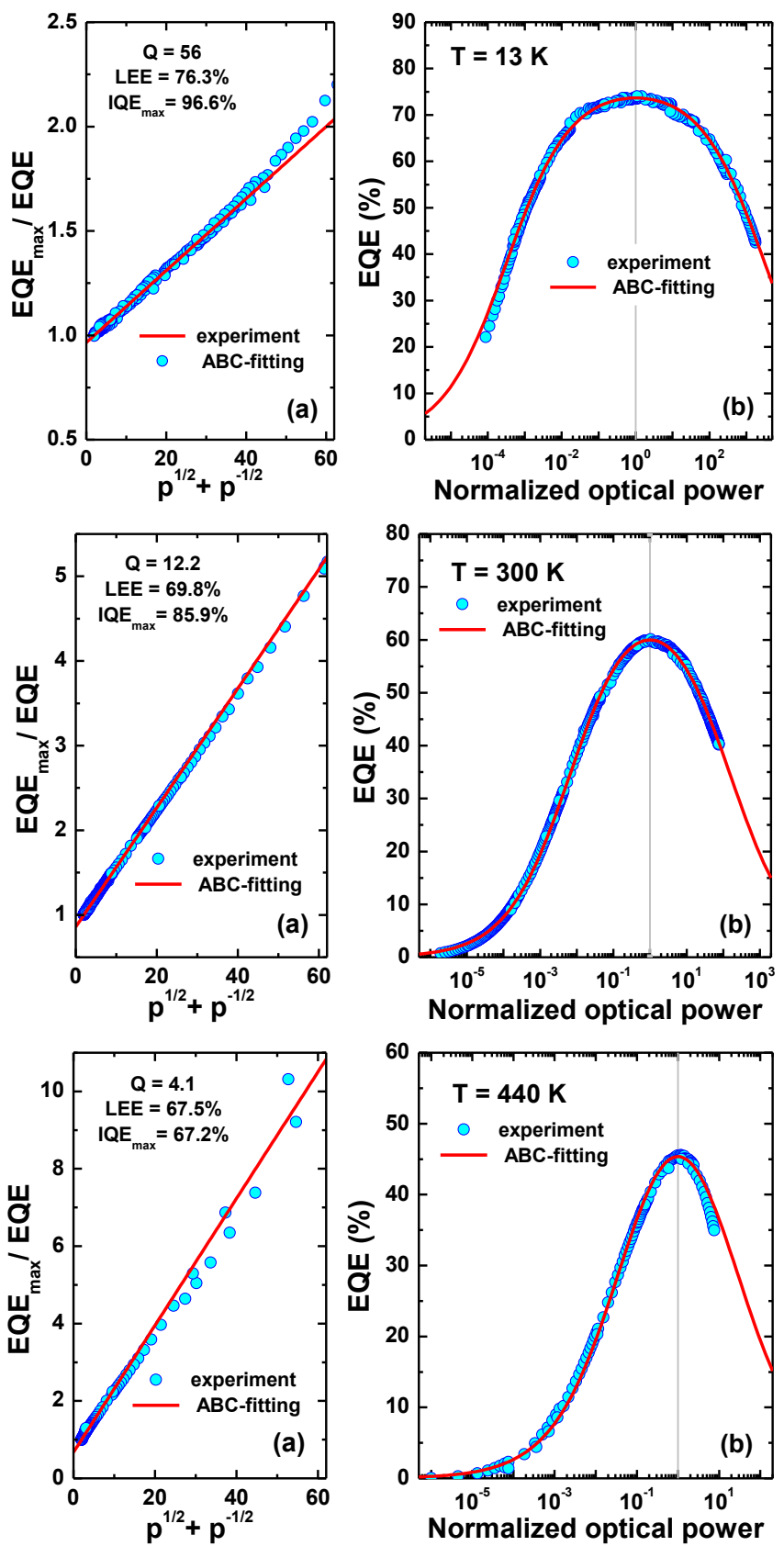

Fig. 5. $\mathrm{EQE}_{\max } / \mathrm{EQE}$ ratio as a function of the $p^{1 / 2}+p^{-1 / 2}$ combination (a) and experimental and theoretical $\mathrm{EQE}$ as a function of the normalized optical power $p$ (b) obtained at various temperatures. Circles indicate experimental points, solid curves are the fittings by ABC-model, using (3) and (2), respectively. $Q$-factors obtained by fitting and corresponding values of LEE and maximum IQE are given in (a). current/optical power, are related to relatively low values of EQE. Since deviation from the linear dependence of the $\eta_{e}^{\max } / \eta_{e}(p)$ ratio on $Y$ become noticeable at $Y>30-40$, the whole $\eta_{e}(p)$ dependence is expected to be fitted well with the ABC-model.

The above conclusion is confirmed by direct simulations of the $\eta_{e}(p)$ dependences, using (2) and the values of LEE obtained by the data processing. Figures $5 \mathrm{~b}$ demonstrate clearly the excellent fitting of the experimental points by the theoretical curves.

The results shown in Fig. 5 are rather typical. Such a data processing has been made for all the temperatures studied. The results of the processing are summarized below in Fig. 6-7.

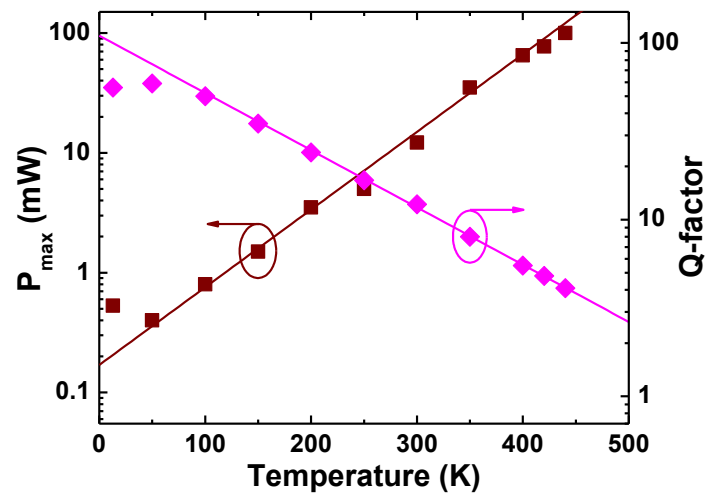

Fig. 6. Optical power $P_{\max }$ corresponding to the EQE maximum and quality factor obtained by fitting procedure. Solid lines are exponential approximations (see text for more detail).

Figure 6 shows the optical power $P_{\max }$ corresponding to the maximum of $\mathrm{EQE}$ obtained from the measurements and the quality factor $Q$ obtained by the fitting procedure described in Sec.IIC. Except for the temperature range of $\sim 13-50 \mathrm{~K}$, both $P_{\max }$ and $Q$-factor are found to vary exponentially with temperature: $P_{\max }=P_{0} \exp \left(T / T_{0}\right)$ and $Q=77 \exp \left(-T / T_{1}\right)$, where $P_{0}=0.17 \mathrm{~mW}, T_{0}=67 \mathrm{~K}$, and $T_{1}=134 \mathrm{~K}$. These approximations are shown in Fig.6 by solid lines. The fact that $T_{1} \cong 2 T_{0}$ means, in particular, that the product $Q^{2} P_{\max }$ is practically independent of temperature, at least in the temperature range of $\sim 70-400 \mathrm{~K}$ where no systematic deviation of the experimental points from the approximations is observed. This fact will be discussed in more detail in the next section.

LEE of the LED obtained by processing is found to depend slightly on temperature (see Fig.7). It decreases from $76.3 \%$ to $67.5 \%$ while the temperature rises from $13 \mathrm{~K}$ to $440 \mathrm{~K}$. Such a relatively low LEE is attributed to the light extraction into the air. Much better, $75-85 \%$ and higher, values of LEE can be achieved in completely packaged LEDs [12].

The extracted peak IQE value drops remarkably with temperature, from $96.6 \%$ at $13 \mathrm{~K}$ to $67.2 \%$ at $440 \mathrm{~K}$. The temperature dependence of the IQE maximum can be approximated by the expression $\eta_{i}^{\max }=Q /(Q+2)$, using the temperature dependence of the $Q$-factor derived from Fig.7. In contrast, IQE corresponding to the operating current 
of $350 \mathrm{~mA}$ is found to be practically independent of temperature except for the high, $T>420 \mathrm{~K}$, temperature range where the peak IQE value approaches that at $350 \mathrm{~mA}$.

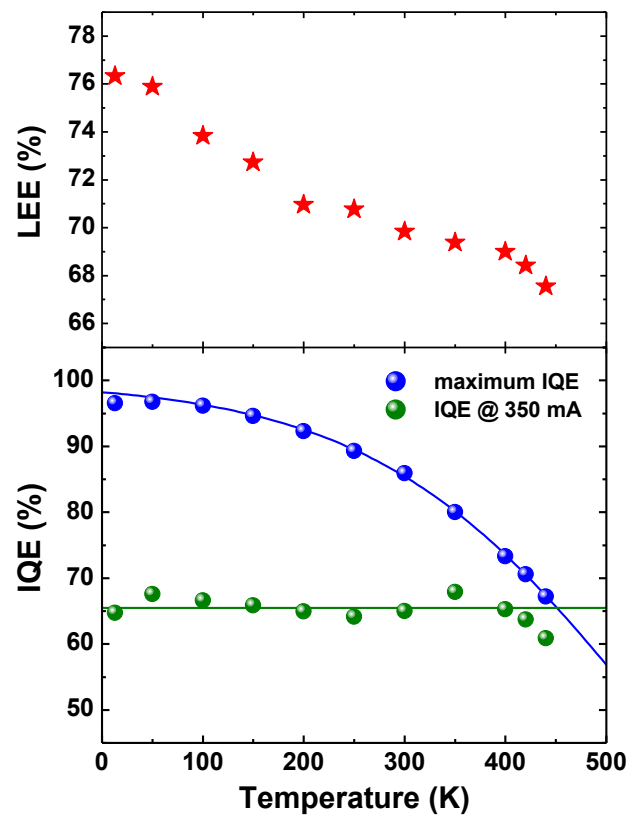

Fig. 7. Light extraction efficiency (top), peak IQE and IQE value at $350 \mathrm{~mA}$ (bottom) obtained by processing the characterization data. Dependence of the peak IQE on temperature is approximated as $\eta_{i}^{\max }=Q /(Q+2)$ with the known temperature dependence of the $Q$-factor (see text). Horizontal line corresponds to the constant value of $65 \%$, approximating the IQE value at $350 \mathrm{~mA}$.

\section{DISCUSSION}

The results of the data processing described in the previous sections are discussed in detail in this section in view of their relevance to LED emission efficiency and underlying mechanisms.

\section{A. Low-current carrier leakage}

As it was discussed in Sec.IIIA, the I-V curves contain typically two sections: the high-current one controlled by the carrier injection in the LED active region and the low-current section tentatively attributed to either trap-assisted tunneling of electrons and holes or carrier leakage through the extended defects like threading dislocations and V-defects. In both latter cases, carriers do not provide any contribution to the radiative recombination, which is confirmed by the absence of a longwavelength peak in the emission spectra (see Sec.IIIB). This means that the low-current sections of the I-V curves correspond to additional carrier losses that are not included in the carrier balance underlying the ABC-model.

Generally, the trap-assisted tunneling followed by the nonradiative carrier recombination can be accounted for by modification of the Shockley-Read-Hall recombination constants [31]. In our case, however, this is not necessary. Indeed, detailed examination of Fig.1 and Fig. 3 shows that the current range of the EQE measurements lies practically always well above the low-current section of the I-V curves corresponding to the trap-assisted tunneling or leakage. In particular, EQE has been measured in the range of $10^{-8}-0.8 \mathrm{~A}$ at $13-50 \mathrm{~K}$, while the low-current section is observable at the currents less than $\sim 10^{-8} \mathrm{~A}$. At $\mathrm{T} \geq 400 \mathrm{~K}$, the low-current section merges with the injection one at $\sim 10^{-4} \mathrm{~A}$, whereas the EQE measurements were carried out at higher currents. This implies that the additional low-current losses of electrons/holes do not interfere the EQE data making reliable their processing by the evaluation procedure based on the ABC-model.

\section{B. Temperature dependence of light extraction efficiency}

LEE obtained by the data processing is found to depend slightly on temperature. This dependence may be explained by considering optical losses inside the LED chip and their dependence on temperature. Generally, there are three channels for the losses: (i) those related to incomplete reflection of the emitted light from the metallic electrodes formed to the contact layers of the LED structure, (ii) those caused by free-carrier absorption in the contact layers, and (iii) the losses related to the band-to-band absorption in the active region of the LED structure.

The optical losses related to incomplete light reflection from the electrodes depend on their reflectance. Using a simple Drude model with the parameters recommended in [32] to approximate the optical constants of silver and accounting for the temperature dependence of the Ag density and its electrical conductivity [33], we have estimated the reflectivity of the GaN/Ag interface corresponding to the normal incidence of light at the wavelength of $450 \mathrm{~nm}$. The reflectivity is found to decrease from $\sim 99 \%$ at $20 \mathrm{~K}$ to $\sim 94 \%$ at $300 \mathrm{~K}$, leading to a rise of the optical losses with temperature.

Free-carrier absorption in the LED contact layers is also temperature-dependent. As a first approximation, the electron concentration in the n-contact layer does not practically depend on temperature due to a high donor concentration resulting in the carrier degeneration. On the other hand, the hole concentration is strongly temperature-dependent because of a high activation energy of the magnesium acceptors. In addition, the mobilities of both electrons and holes decrease substantially with temperature. Since the free-carrier absorption cross-section is roughly inversely dependent on the carrier mobility, the temperature rise leads to enhancement of this process, increasing the losses of emitted photons.

The optical losses caused by the band-to-band absorption of emitted light in the InGaN QWs depend largely on the red shift of the LED emission spectrum from the absorption edge, being dependent on temperature as well. According to the data reported in [34] for the LED emitting at $400 \mathrm{~nm}$, such a shift of $\sim 35 \mathrm{meV}$ is observed at low, $\sim 20-70 \mathrm{~K}$, temperature and then it reduces dramatically, vanishing at $\sim 230-250 \mathrm{~K}$. The shift reduction with temperature should be accompanying by an increase of the band-to-band light absorption.

Therefore, all the above mechanisms are expected to increase the optical losses inside the LED die at elevated temperatures. This leads eventually to reduction of the chip LEE, in line with the trend displayed in Fig.7. 


\section{Temperature dependence of recombination constants}

The measurements of EQE as a function of current/output optical power do not allow separate evaluation of the recombination constant $A, B$, and $C$. For this purpose, such experiments should be supplemented with additional ones, e. g. like those measuring the carrier differential life time versus current [35-37]. Nevertheless, some of the results obtained in our study enable making a certain guess on the temperature dependence of the recombination constants.

It has been already mentioned in Sec.IIID, that the product $Q^{2} P_{\max }$ is rather weakly dependent of temperature in the range of $\sim 70-400 \mathrm{~K}$. According to the ABC-model, the parameter $P_{\text {max }}=\hbar \omega \eta_{\text {ext }} V_{r}(A B / C)$ with $V_{r}$ being the volume of the active region where carrier recombination effectively occurs. Correspondingly, the product $Q^{2} P_{\max }=\hbar \omega \eta_{e x t} V_{r}\left(B^{3} / C^{2}\right)$ does not contain the recombination constant $A$ at all. The single parameters $Q^{2}$ and $P_{\max }$ vary by more than two orders of magnitude in the temperature range of 70-400 K (Fig.6). To understand the possible contribution of particular recombination constants to the temperature dependence of the parameters $Q^{2}$ and $P_{\max }$, let us assume $V_{r} \propto T^{k}, B \propto T^{\beta}$, and $C \propto T^{\gamma}$. Such a power-law approximations are evidently valid for the recombination constant $B$ and, in some cases of non-threshold Auger processes, for the recombination constant $C[38,39]$. For the recombination volume $V_{r}$, however, the power-law dependence can be considered only as an estimate roughly accounting for the full range of the parameter variation with temperature. Then the independence of the product $Q^{2} P_{\text {max }}$ on temperature is equivalent to the following relation between the power coefficients: $k+3 \beta=2 \gamma$.

Regarding the available data on the non-uniform carrier injection in MQW LED structures [17,40,41], we can conclude that the recombination volume $V_{r}$ may vary between that of a single QW and the total volume of all the QWs in the MQW active region. This limits the coefficient $k$ in the assumed temperature dependence of $V_{r}$ by the value of $\sim 1$ (the value $k=0$ corresponds to a temperature independent $V_{r}$ ). In the case of a thin $\mathrm{QW}$ confining the ground electron and hole states only, the coefficient $\beta=-1$. So, the coefficient $\gamma$ may vary between -1 (for $k=0$ ) and -1.5 (for $k=1$ ) in accordance with the above relationship. This means eventually that both recombination coefficients $B$ and $C$ should descend with temperature.

A similar result has been recently reported by Hader et al. [42]. They referred to the data of [30] where the normalized EQE of a blue LED fabricated by Nichia was measured vs. current at different temperatures. To analyze the behavior of the recombination constants, the authors of [42] calculated theoretically the dependence $B(T)$ and then extracted the constants $A$ and $C$ by fitting the data of [30] by the ABCmodel. The results of the calculations and data processing were approximated as follows: $B \propto T^{-7 / 4}$ and $C \propto T^{-5 / 2}$ (or $T^{-3}$ ) [42]. In this case, both $B$ and $C$ constants were descending functions of temperature. Moreover, using the above approximations, we have obtained the product $Q^{2} P_{\max }$ to be proportional to $T^{-1 / 4}$ (or $T^{+1 / 8}$ ) for the temperature independent recombination volume. The latter estimate shows the product to be actually independent of temperature within the accuracy of the employed approximations.

The descending temperature dependence of the recombination constant $C$ does not correlate with available theoretical considerations [43-45] of the Auger recombination in bulk InGaN and QWs (in particular, a weak increase of the $C$ constant with temperature has been predicted in [43] and [45]). This fact was used in [42] as the basis for suggesting a mechanism of the efficiency droop alternative to that involving Auger recombination. Leaving aside the discussion on the droop mechanisms, we would like to mention that the descending temperature dependence of the Auger coefficient has been already reported, e. g., for $4 \mathrm{H}-\mathrm{SiC}$ heavy-doped with acceptors [46] and also predicted theoretically for some nonthreshold Auger processes in low-dimensional structures [38,39].

It follows from the above discussion that our results on the temperature dependence of the recombination constants $B$ and $C$ agree well with those reported in [42] and based on the observations made in [30]. On the other hand, they are in some conflict with the constants behavior reported in [36] for the temperature range of $300-425 \mathrm{~K}$. In that study, the recombination constants were determined from the joint fitting the EQE and differential life time of SQW blue LEDs grown on silicon substrates. The data obtained provided descending dependence $B(T)$ with $\beta \approx-1$ and ascending dependence $C(T)$ with $\gamma \approx+1.2$. A possible explanation for the discrepancy between our results and those reported in [36] may be as follows. Our consideration attributes the recombination volume variation to the whole temperature range of $\sim 70-400$ $\mathrm{K}$. In practice, $V_{r}$ may vary in a much narrower range, being nearly constant at other temperatures. This would result in a change of the power coefficient for the temperature dependence of the Auger recombination constant, including possible switching from descending to ascending function. Such an opportunity is in line with particular calculations presented in [38,39] for the QWs and quantum dots made of conventional III-V compounds. Being justified experimentally for III-nitride QWs, the changes of the power coefficient of the temperature-dependent recombination constant $C$ would be the evidence for a non-threshold character of the Auger processes occurring in the LED heterostructures.

\section{CONCLUSION}

The paper reports on temperature variation of electrical and optical characteristics of a high-brightness blue LED measured in a wide range of 13-440 $\mathrm{K}$. The suggested procedure of the data processing based on the ABC-model has enabled separate estimating of epi-structure IQE and LEE of the packaged LED chip. To our knowledge, such a detailed study of the emission efficiency and its temperature dependence is reported for the first time.

We have found the ABC-model to fit well the LED emission efficiency in the whole range of the temperature 
variation studied here. The low-current carrier losses tentatively attributed to either trap-assisted tunneling or to carrier leakage along extended defects did not interfere the results of the efficiency measurements. Estimations have shown LEE to decrease slightly with temperature, from $\sim 76 \%$ at $13 \mathrm{~K}$ to $\sim 68 \%$ at $440 \mathrm{~K}$. The peak IQE value is found to be $\sim 86 \%$ at room temperature, increasing up to $\sim 96-97 \%$ at low temperatures of about $13-50 \mathrm{~K}$. The latter justifies the use of the temperature-dependent EL measurements for estimating IQE of blue LEDs. In contrast, high-current IQE value corresponding to the operating current of $350 \mathrm{~mA}$ has turned out to be practically independent of temperature and equal to $\sim 65 \%$.

The latter fact is in line with the temperature-independent product of the parameters $Q^{2} P_{\max }$ derived from the data obtained. Assuming a monotonic power-law temperature dependence of the recombination volume and radiative and Auger recombination constants $B$ and $C$ we came to conclusion that both $B$ and $C$ constant should descend with temperature. The conclusion does not correlate with available theoretical models of Auger recombination in III-nitride materials. A possible explanation of the above discrepancy given in the paper implies a non-monotonic temperature dependence of the Auger recombination coefficient, which may be the evidence for a non-threshold character of the Auger processes occurring in the LED structure. More efforts, aimed primarily at direct measurements of the recombination coefficients in a wide temperature range, is required in future to clarify finally this aspect.

We believe that the proposed procedure of data processing could be also helpful for optimization of the LED structure and chip design, as well as for further studying the mechanisms responsible for the efficiency droop and for the efficiency 'green gap' still remaining a major problem for solid-state lighting.

\section{REFERENCES}

[1] T. Mukai, M. Yamada, and S. Nakamura, "Characteristics of InGaNBased UV/Blue/Green/Amber/Red Light-Emitting Diodes," Jpn. J. Appl. Phys., vol. 38, no. 7A, pp. 3976-3981, Apr., 1999.

[2] M. R. Krames, G. Christenson, D. Collins, L. W. Cook, M. G. Craford, A. Edwards, R. M. Fletcher, N. F. Gardner, W. K. Goetz, W. R. Imler, E. Johnson, R. S. Kern, R. Khare, F. A. Kish, C. Lowery, M. J. Ludowise, R. Mann, M. Maranowski, S. A. Maranowski, P. S. Martin, J. O'Shea, S. L. Rudaz, D. A. Steigerwald, J. Thompson, J. J. Wierer, J. Yu, D. Basile, Ying-Lan Chang, G. Hasnain, M. Heuschen, K. P. Killeen, C. P. Kocot, S. Lester, J. N. Miller, G. O. Mueller, R. MuellerMach, S. J. Rosner, R. P. Schneider, Jr., T. Takeuchi, and T. S. Tan, "High-brightness AlGaInN light-emitting diodes, " Proc. SPIE, vol. 3938, pp. 2-12, Apr., 2000.

[3] G. Verzellesi, D. Saguatti, M. Meneghini, F. Bertazzi, M. Goano, G. Meneghesso, and E. Zanoni, "Efficiency droop in $\mathrm{InGaN} / \mathrm{GaN}$ blue light-emitting diodes: Physical mechanisms and remedies," J. Appl. Phys., vol. 114, no. 7, pp. 071101, Aug., 2013.

[4] Y. C. Shen, G. O. Mueller, S. Watanabe, N. F. Gardner, A. Munkholm, and M. R. Krames, "Auger recombination in $\mathrm{InGaN}$ measured by photoluminescence," Appl. Phys. Lett., vol. 91, no. 14, pp. 141101, Oct., 2007.

[5] K. A. Bulashevich and S. Yu. Karpov, "Is Auger recombination responsible for the efficiency rollover in III-nitride light-emitting diodes?," Phys. Stat. Solidi C, vol. 5, no. 6, pp. 2066-2069, March, 2008.

[6] A. Laubsch, M. Sabathil1, W. Bergbauer, M. Strassburg, H. Lugauer, M. Peter, S. Lutgen, N. Linder, K. Streubel, J. Hader, J. V. Moloney, B.
Pasenow, and S. W. Koch, "On the origin of IQE-'droop' in InGaN LEDs,” Phys. Stat. Solidi C, vol. 6, no. S2, pp. S913-S916, June, 2009.

[7] J. Iveland, L. Martinelli, J. Peretti, J. S. Speck, and C. Weisbuch, "Direct Measurement of Auger Electrons Emitted from a Semiconductor LightEmitting Diode under Electrical Injection: Identification of the Dominant Mechanism for Efficiency Droop," Phys. Rev. Lett., vol. 110, no. 17, pp. 177406, Apr., 2013.

[8] M. Binder, A. Nirschl, R. Zeisel, T. Hager, H.-J. Lugauer, M. Sabathil, D. Bougeard, J. Wagner and B. Galler, "Identification of $n n p$ and $n p p$ Auger recombination as significant contributor to the efficiency droop in (GaIn)N quantum wells by visualization of hot carriers in photoluminescence," Appl. Phys. Lett., vol. 103, no. 7, pp. 071108, Aug., 2013.

[9] B. Galler, H.-J. Lugauer, M. Binder, R. Hollweck, Y. Folwill, A. Nirschl, A. Gomez-Iglesias, B. Hahn, J. Wagner, and M. Sabathil, "Experimental Determination of the Dominant Type of Auger Recombination in InGaN Quantum Wells," Appl. Phys. Express, vol. 6, no. 11, pp. 112101, Oct., 2013.

[10] M. V. Bogdanov, K. A. Bulashevich, O. V. Khokhlev, I. Yu. Evstratov, M. S. Ramm, and S. Yu. Karpov, "Current crowding effect on light extraction efficiency of thin-film LEDs," Phys. Stat. Solidi C, vol. 7, no. 7-8, pp. 2124-2126, May, 2010.

[11] O. B. Shchekin, J. E. Epler, T. A. Trottier, T. Margalith, D. A. Steigerwald, M. O. Holcomb, P. S. Martin, and M. R. Krames, "High performance thin-film flip-chip InGaN-GaN light-emitting diodes," Appl. Phys. Lett., vol. 89, no. 7, pp. 071109, Aug., 2006.

[12] A. Laubsch, M. Sabathil, J. Baur, M. Peter, and B. Hahn, "High-Power and High-Efficiency InGaN-Based Light Emitters," IEEE Trans. Electron. Devices, vol. 57, no. 1, pp. 79-87, Jan., 2010.

[13] A. I. Zhmakin, "Enhancement of light extraction from light emitting diodes", Phys. Rep., vol. 498, no. 4-5, pp. 189-241, Nov., 2010.

[14] S. Watanabe, N. Yamada, M. Nagashima, Y. Ueki, C. Sasaki, Y. Yamada, T. Taguchi, K. Tadatomo, H. Okagawa, and H. Kudo, "Internal quantum efficiency of highly-efficient $\mathrm{In}_{\mathrm{x}} \mathrm{Ga}_{1-\mathrm{x}} \mathrm{N}$-based near-ultraviolet light-emitting diodes," Appl. Phys. Lett., vol. 83, no. 24, pp. 4906-4908, Oct., 2003.

[15] A. Hangleiter, D. Fuhrmann, M. Grewe, F. Hitzel, G. Klewer, S. Lahmann, C. Netzel, N. Riedel, and U. Rossow, "Towards understanding the emission efficiency of nitride quantum wells," Phys. Stat. Solidi A, vol. 201, no. 12, pp. 2808-2813, July, 2004.

[16] G. Chen, M. Craven, A. Kim, A. Munkholm, S. Watanabe, M. Camras, W. Götz, and F. Steranka, "Performance of high-power III-nitride light emitting diodes," Phys. Stat. Solidi A, vol. 205, no. 5, pp. 1086-1092, March, 2008

[17] M. Peter, A. Laubsch, W. Bergbauer, T. Meyer, M. Sabathil, J. Baur, and B. Hahn, "New developments in green LEDs," Phys. Stat. Solidi A, vol. 206, no. 6, pp. 1125-1129, Jan., 2009.

[18] Han-Youl Ryu, Hyun-Sung Kim, and Jong-In Shim, "Rate equation analysis of efficiency droop in InGaN light-emitting diodes," Appl. Phys. Lett., vol. 95, no. 8, pp. 081114, Aug., 2009.

[19] Qi Dai, Qifeng Shan, Jaehee Cho, E. F. Schubert, M. H. Crawford, D. D. Koleske, Min-Ho Kim, and Yongjo Park, "On the symmetry of efficiency-versus-carrier-concentration curves in $\mathrm{GaInN} / \mathrm{GaN}$ lightemitting diodes and relation to droop-causing mechanisms," Appl. Phys. Lett., vol. 98, no. 3, pp. 033506, Jan., 2011.

[20] Guan-Bo Lin, Qifeng Shan, A. J. Birkel, Jaehee Cho, E. F. Schubert, M. H. Crawford, K. R. Westlake, and D. D. Koleske, "Method for determining the radiative efficiency of GaInN quantum wells based on the width of efficiency-versus-carrier-concentration curve," Appl. Phys. Lett., vol. 101, no. 24, pp. 241104, Dec., 2012.

[21] P. Kivisaari, L. Riuttanen, J. Oksanen, S. Suihkonen, M. Ali, H. Lipsanen, and J. Tulkki, "Electrical measurement of internal quantum efficiency and extraction efficiency of III-N light-emitting diodes," Appl. Phys. Lett., vol. 101, no. 2, pp. 021113, July, 2012.

[22] C. van Opdorp and G. W. 't Hooft, "Method for determining effective non radiative lifetime and leakage losses in double-heterostructure lasers," J. Appl. Phys., vol. 52, no. 6, pp. 3827-3839, Feb., 1981.

[23] M. Meneghini, N. Trivellin, G. Meneghesso, E. Zanoni, U. Zehnder, and B. Hahn, "A combined electro-optical method for the determination of the recombination parameters in InGaN-based light-emitting diodes," $J$. Appl. Phys., vol. 106, no. 11, pp. 114508, Dec., 2009.

[24] S. Yu. Karpov, "Effect of localized states on internal quantum efficiency of III-nitride LEDs," Phys. Stat. Solidi RRL, vol. 4, no. 11, pp. 320-322, Sept., 2010. 
[25] Qi Dai, Qifeng Shan, Jing Wang, S. Chhajed, Jaehee Cho, E. F. Schubert, M. H. Crawford, D. D. Koleske, Min-Ho Kim, and Yongjo Park, "Carrier recombination mechanisms and efficiency droop in GaInN/GaN light-emitting diodes," Appl. Phys. Lett., vol. 97, no. 13, pp. 133507, Sept., 2010.

[26] A. Laubsch, M. Sabathil, J. Baur, M. Peter, and B. Hahn, "High-Power and High-Efficiency InGaN-Based Light Emitters," IEEE Trans. Electron. Dev., vol. 57, no. 1, pp. 79-87, Dec., 2010.

[27] K. A. Bulashevich, O. V. Khokhlev, I. Yu. Evstratov, and S. Yu. Karpov, "Simulation of light-emitting diodes for new physics understanding and device design," Proc. SPIE, vol. 8278, pp. 827619-112, Feb., 2012.

[28] P. G. Eliseev, M. Osin'ski, Hua Li, and Irina V. Akimova, "Recombination balance in green-light-emitting GaN/InGaN/AlGaN quantum wells," Appl. Phys. Lett., vol. 75, no. 24, pp. 3838-3840, Dec., 1999

[29] Yong-Hoon Cho, G. H. Gainer, A. J. Fischer, J. J. Song, S. Keller, U. K. Mishra, and S. P. DenBaars, "S-shaped" temperature-dependent emission shift and carrier dynamics in InGaN/GaN multiple quantum wells," Appl. Phys. Lett., vol. 73, no. 10, pp. 13701373, Sept., 1998.

[30] K. Fujiwara, H. Jimi, and K. Kaneda, "Temperature-dependent droop of electroluminescence efficiency in blue (In, Ga)N quantum-well diodes," Phys. Stat. Solidi C, vol. 6, no. S2, pp. S814-S817, Jan., 2009.

[31] K. Sakowski, L. Marcinkowski, S. Krukowski, S. Grzanka, and E. Litwin-Staszewska, "Simulation of trap-assisted tunneling effect on characteristics of gallium nitride diodes," Appl. Phys. Lett., vol. 111, no. 12, pp. 123115, June, 2012.

[32] C. Oubre and P. Nordlander, "Optical Properties of Metallodielectric Nanostructures Calculated Using the Finite Difference Time Domain Method," J. Phys. Chem. B, vol. 108, no. 17, pp. 17740-17747, Sept., 2004

[33] D. B. Tanner and D. C. Larsen, "Electrical Resistivity of Silver Films," Phys. Rev., vol. 166, no. 3, pp. 652-655, March, 1968.

[34] C. S. Kim, J. H. Kim, K. J. Yee, H. K. Kwon, H. S. Lee, J. S. Park, and K. J. Yee, "Temperature- and Bias-dependent Study of Photocurrent Spectroscopy in an InGaN Light-emitting Diode Operating near 400 nm,” J. Korean Phys. Soc., vol. 57, no. 4, pp. 793-796, Oct., 2010.

[35] A. David and M. J. Grundmann, "Droop in InGaN light-emitting diodes: A differential carrier lifetime analysis," Appl. Phys. Lett., vol. 96, no. 10, pp. 103504, March, 2010.

[36] B. Galler, P. Drechsel, R. Monnard, P. Rode, P. Stauss, S. Froehlich, W. Bergbauer, M. Binder, M. Sabathil, B. Hahn, and J. Wagner, "Influence of indium content and temperature on Auger-like recombination in InGaN quantum wells grown on (111) silicon substrates," Appl. Phys. Lett., vol. 101, no. 13, pp. 131111, Sept., 2012.

[37] D. Schiavon, M Binder, M. Peter, B. Galler, P. Drechsel, and F. Scholz, "Wavelength-dependent determination of the recombination rate coefficients in single-quantum-well GaInN/GaN light emitting diodes," Phys. Stat. Solidi B, vol. 250, no. 2, pp. 283-290, Nov., 2012.

[38] A. S. Polkovnikov and G. G. Zegrya, "Auger recombination in semiconductor quantum wells," Phys. Rev. B, vol. 59, no. 7, pp. 40394056, Feb., 1998.

[39] G. G. Zegrya and D. M. Samosvat, "Mechanisms of Auger Recombination in Semiconducting Quantum Dots," J. Experimental and Theoretical Physics, vol. 104, no. 6, pp. 951-965, June, 2007.

[40] A. David,_ M. J. Grundmann, J. F. Kaeding, N. F. Gardner, T. G. Mihopoulos, and M. R. Krames, "Carrier distribution in (0001)InGaN/GaN multiple quantum well light-emitting diodes," Appl. Phys. Lett., vol. 92, no. 5, pp. 053502, Feb., 2008.

[41] B. Galler, A. Laubsch, A. Wojcik, H. Lugauer, A. Gomez-Iglesias, M. Sabathil, and B. Hahn, "Investigation of the carrier distribution in InGaN-based multi-quantum-well structures," Phys. Stat. Solidi C, vol. 8, no. 7-8, pp. 2372-2374, Nov., 2011.

[42] J. Hader, J. V. Moloney, and S. W. Koch, "Temperature-dependence of the internal efficiency droop in GaN-based diodes," Appl. Phys. Lett., vol. 99, no. 18, pp. 181127, Nov., 2011.

[43] E. Kioupakis, P. Rinke, K. T. Delaney, and C. G. Van de Walle, "Indirect Auger recombination as a cause of efficiency droop in nitride light-emitting diodes," Appl. Phys. Lett., vol. 98, no. 16, pp. 161107, Apr., 2011.

[44] R. Vaxenburg, E. Lifshitz, and Al. L. Efros, "Suppression of Augerstimulated efficiency droop in nitride-based light emitting diodes," Appl. Phys. Lett., vol. 102, no. 3, pp. 031120, Jan., 2013.
[45] F. Bertazzi, X. Zhou, M. Goano, G. Ghione, and E. Bellotti, "Auger recombination in InGaN/GaN quantum wells: A full-Brillouin-zone Study," Appl. Phys. Lett., vol. 103, no. 8, pp. 081106, Aug., 2013.

[46] A. Galeckas, J. Linnros, V. Grivickas, U. Lindefelt, and C. Hallin, "Auger recombination in 4H-SiC: Unusual temperature behavior," Appl. Phys. Lett., vol. 71, no. 22, pp. 3269-3271, Oct., 1997.

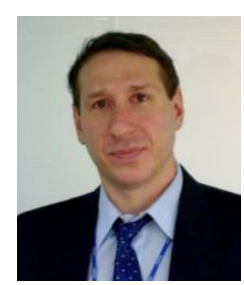

Ilya E. Titkov received his $\mathrm{PhD}$ degree in 22 November 2001 from Saint-Petersburg State Polytechnical University, department of semiconductor physics and nanostructures. Since then, his previous positions include: Samsung LED - 2009-2012 Senior engineer; Samsung Electro-Mechanics 2008-2009 Senior engineer; Researcher at the Ioffe Institute from 2001 to 2008. Dr. Titkov has been awarded with seven personal grants and fellowships (Soros, INTAS, RFBR etc.). In 2006-2007, he obtained the Russian President's Foundation personal grant for young Ph.D researchers in the field of laser ablation for $\mathrm{ZnO} / \mathrm{GaN}$ based LEDs. He is co-author of more than 30 publications and 1 patent with totally 111 citations. Dr. Titkov is currently working on novel LED materials and devices as part of the FP7 European IP project (NEWLED) in the School of Engineering and Applied Science, Aston University, Birmingham, UK.

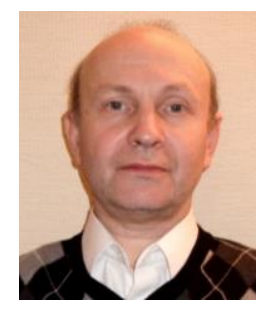

Sergey Yu. Karpov has received his Ph.D. degree from the A. F. Ioffe Physico-Technical Institute RAS (St. Petersburg, Russia) in 1982. He worked at the Ioffe Institute in the period 1977-1991 and at the Advanced Technology Center in 1991-1998. Since 1998 , he is working as a senior researcher at STR Group - Soft-Impact, Ltd. He is the co-author/author of more than 260 publications in the peerreviewed scientific journals and books and of 12 Russian and 10 US patents. His scientific interests are focused on materials science, physics of semiconductors, and modeling/simulation of crystal growth, epitaxy, and optoelectronic devices.

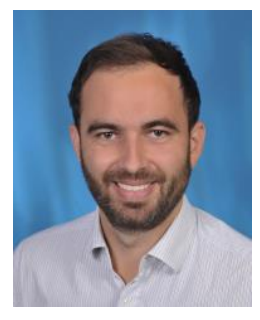

Bastian Galler received his diploma in physics from the Technical University of Munich in 2008. His thesis was carried out at the Walter-Schottky-Institute and covers electrically detected magnetic resonance studies of phosphorous donors at the $\mathrm{Si} / \mathrm{SiO} 2$ interface. Afterwards, he joined the R\&D department of OSRAM Opto Semiconductors for a $\mathrm{PhD}$ project (in cooperation with the Fraunhofer Institute for Applied Solid-State Physics) investigating carrier recombination and transport in (AlGaIn)N-based light-emitting diodes. He is now a Senior Scientist focusing on the further understanding and improvement of internal quantum efficiency. 


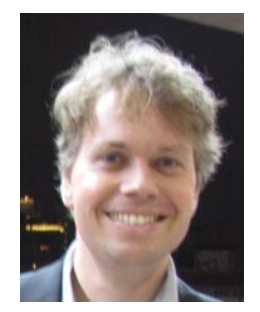

Martin Strassburg achieved his Ph.D. in Semiconductor Physics at the Technical University of Berlin in 2002. From 2003 2005 he received the Feodor-Lynenfellowship of the Humboldt-foundation to pursue research on the development of group-III nitride materials for solid state lighting, high power electronics, solar cell and spintronics applications at Georgia Institute of Technology in Atlanta, USA. In 2005 he joined OSRAM Opto Semiconductors $\mathrm{GmbH}$. Since 2006 he was responsible for the Nitride MOVPE development in the Advanced Concepts \& Engineering department. Since 2012, he is heading the open innovation activities and is responsible for the technology innovation. He holds numerous patents in this area and coauthored more than 100 papers on material development for optoelectronic applications.

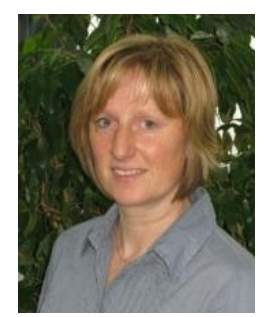

Ines Pietzonka received her Ph.D. at the University of Leipzig (Germany) in 1999 with focus on epitaxial growth of GaInP/GaAs structures. From 1999-2001 she worked as Marie-Curie-fellow on the development of group-III-V nanostructures for various material systems such as GaN/GaAs-, GaP/GaAs- and Ge/Si at the University of Lund (Sweden) under Prof. L. Samuelson and Prof. W. Seifert. In 2001 she joined Osram Opto Semiconductors $\mathrm{GmbH}$ as development engineer and is responsible for the development of new epitaxial structures for AlGaInP-and GaInN-based LEDs as well as for AlGaAs/InGaAs-based lasers. She holds several patents and authored and/or co-authored numerous papers in this field.

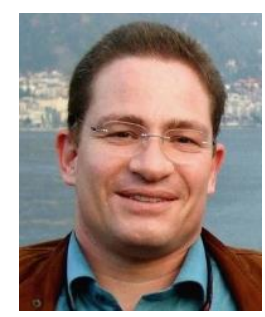

Hans-Jürgen Lugauer received the $\mathrm{PhD}$ degree in physics in 1999 from the University of Würzburg, Germany. During this time he significantly contributed to the development of BeZnCdSe based green laser diodes.

From 1996 to 1999 he was Managing Director of the Bavarian Research Cooperation for Optoelectronics (Forschungsverbund Optoelektronik FOROPTO). In 1999, he joined the R\&D department of OSRAM Opto Semiconductors $\mathrm{GmbH}$ in Regensburg, Germany. As Senior Key Expert he was responsible for the development of AlInGaN based light emitting devices with focus on the optimization of the metalorganic vapour phase epitaxy (MOVPE) growth processes. He is coauthor or author of more than 70 publications and currently holds 53 patents in this area. Since 2010 he heads the "Novel Technologies" group within the Advanced Concepts \& Engineering department of OSRAM Opto Semiconductors, focusing on the development of novel devices, processes and materials for optoelectronic components.

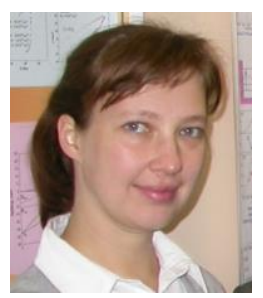

Vera L. Zerova received the $\mathrm{PhD}$ degree in Physics of Semiconductors from St. Petersburg State Polytechnic University, Department of Semiconductor Physics and Nanoelectronics, in 2006. Her thesis was devoted to optical phenomena in quantum wells based on III-V compound semiconductors. Her research interests include optical phenomena and non-equilibrium charge carriers in semiconductor nanostructures, physics of semiconductor lasers and light emitted diodes, modeling and simulation, quantum mechanical calculations. Presently she is a volunteer researcher with the School of Engineering and Applied Science, Aston Institute of Photonic Technologies, Aston University, Birmingham, UK.

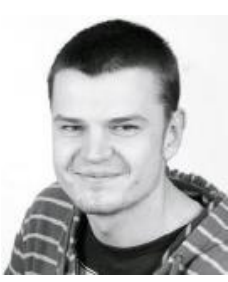

Modestas Zulonas received the B.S. degree in general physics from Vilnius University, Physics faculty, Vilnius, Lithuania, in 2009 and the M. S. degree in solid state physics, Vilnius University in 2011. He is currently pursuing the Ph.D. degree in School of Engineering and Applied Science, Aston Institute of Photonic Technologies, Aston University, Birmingham, UK. From 2008 to 2009, he was a research assistant with the Vilnius University, physics faculty, radiophysics division. His research interest was 1/f noise in high power light emitting diodes and he write Bachelor thesis. Also from 2009 to 2011, he was a research assistant with the Vilnius University, solid state physics division. His research interest was in chemical solution deposition, spin coating, thermal evaporating and organic thin film field effect transistors. He write Master's thesis. His Ph.D. research is international EU project based on the study of InGaN based white, blue, green colour light emitting diodes, including synthesis, properties, applications.

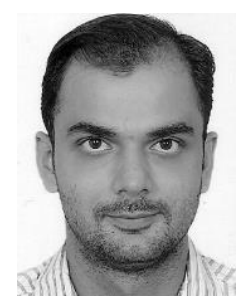

Amit Yadav was born in Delhi, India. He received his $\mathrm{B}$. Tech. in computer science and engineering from GGSIP University, Delhi, India in 2006 and M.Sc. in electronics and electrical engineering from University of Glasgow, Glasgow, UK in 2009. He is currently working towards the $\mathrm{PhD}$ degree in the School of Engineering and Applied Science, Aston Institute of Photonic Technologies, Aston University, Birmingham, UK. His research interests include development and characterization of electronic and optoelectronic devices based on III-V materials. His current research focuses on electrical and optical characterization for III-nitride based optoelectronic devices such as LEDs and Laser diodes.

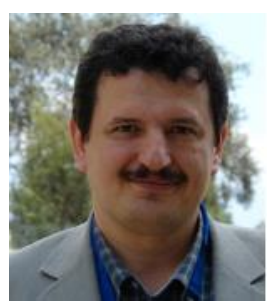

Edik U. Rafailov (SM'05) received the Ph.D. degree from the Ioffe Institute, St Petersburg. In 1997 he moved from St Petersburg to St Andrews University as a Research Fellow and in 2005 he moved to Dundee University and established new Photonics and Nanoscience Group. In 
2014 he moved to Aston University. He has authored and coauthored over 350 articles in referred journals and conference proceedings, including two books, five invited chapters and numerous invited talks to SPIE, CLEO and LEOS. He also holds $10 \mathrm{UK}$ and two US patents. Prof. Rafailov coordinated a $€ 14.7 \mathrm{M}$ FP7 European IP project (FAST-DOT) intended to develop new miniature low-cost ultrafast lasers based on quantum-dot materials for applications in Biophotonics and cellular surgery. Currently, he coordinated the $€ 11.8 \mathrm{M}$ FP7 European IP project (NEWLED) project aims to develop a new generation of white light-emitting LED lights, which would be much more efficient than existing light bulbs. He also leads a few others projects funded by FP7 EU and EPSRC. His current research interests include novel highpower $\mathrm{CW}$, ultrashort-pulse and high-repetition rate lasers and LEDs; generation of UV/visible/IR/MIR and $\mathrm{THz}$ radiation, nanostructures; nonlinear and integrated optics; Biophotonics. 